Methods: We treated 37 RA patients with either etanercept (ETN) or certolizumab pegol (CZP) in a 12 month follow-up study. Assessments were performed at baseline, and 3, 6 and 12 months after treatment initiation. Serum chemerin and adiponectin concentrations were measured by commercially available ELISA kits (R and D System, MN and USA). PON1 and arylesterase activities were measured by spectrophotometry. In addition, age, disease duration, disease activity (DAS28), CRP, anti-CCP, IgM rheumatoid factor and plasma lipid levels were also assessed. Arterial flow-mediated vasodilation (FMD), carotid intima-media thickness (cIMT) and arterial pulse-wave velocity (PWV) were assessed by ultrasound.

Results: Anti-TNF treatment resulted in a significant decrease in the levels of chemerin $(p<0.001)$ and adiponectin $(p<0.007)$ after 12 months. There were no significant changes in the levels of other metabolic biomarkers. We found the following correlations between the baseline values: the PON1 levels correlated with the age of patients $(R=0.466, p=0.004)$. The adiponectin correlated with the disease activity $(R=0.385, p=0.030)$, HDL-C $(R=0.417, p=0.012)$ and the triglyceride levels $(R=0.481, p=0.003)$. The total cholesterol correlated with the PWV $(R=0.449, p=0.021)$ and the levels of the LDL-C $(R=0.911, p<0.001)$. The baseline triglyceride correlated with the $\operatorname{lgM}$ rheumatoid factor $(R=0.343$, $p=0.021)$; and the levels of LDL-C correlated with the PWV values $(R=0.444$, $\mathrm{p}=0.023)$.

Conclusions: Metabolic factors, such as certain adipokines, PON1 and arylesterase may play a role in oxidative stress and atherosclerosis associated with RA. Anti-TNF treatment may affect adipokine levels.

Disclosure of Interest: None declared

DOI: 10.1136/annrheumdis-2018-eular.2122

\section{AB0384 RHEUMATIC ARTHRITIS PATIENTS WITH VERY HIGH CARDIOVASCULAR RISK: POOR RESULTS IN CHANGES IN LIFESTYLE DURING FOLLOW-UP}

N. Vegas-Revenga ${ }^{1}$, V. Portilla ${ }^{1}$, L.C. Domínguez-Casas ${ }^{1}$, J.L. Martín-Varillas ${ }^{1}$, B. Atienza-Mateo ${ }^{1}$, M. Agudo ${ }^{1}$, J. Rueda-Gotor ${ }^{1}$, J.L. Hernández ${ }^{2}$, R. Blanco ${ }^{1}$, M. A. González-Gay ${ }^{1}$, A. Corrales ${ }^{1} .{ }^{1}$ Rheumatology; ${ }^{2}$ Internal Medicine, Hospital Universitario Marqués de Valdecilla, Santander, Spain

Background: Rheumatoid arthritis (RA) is associated with accelerated atherosclerosis, which results in high cardiovascular morbidity and mortality. The European Atherosclerosis Society recommends a strict control of cardiovascular (CV) risk factors, focusing in a healthy lifestyle, for the prevention of future CV events. Objectives: Our aim was to assess the characteristics of healthy lifestyle in patients with RA and very high risk of CV events.

Methods: Prospective study that included a group of 113 patients, of whom 65 presented carotid plaques on carotid ultrasound study and, due to this, were categorised as having very CV high risk at the baseline visit. At this time, patients were informed about the risks associated to their disease and the high risk of having $\mathrm{CV}$ events. They were even warned of the need of healthy lifestyle habits.

Data on lifestyle, smoking, obesity and diabetes mellitus were collected at baseline and last visit (with an interval of more than 5 years)

Abstract AB0384 - Table 1. Clinic characteristics of 65 patients with RA and very high CV risk.

\begin{tabular}{|l|c|}
\hline \multicolumn{1}{|c|}{ CLINIC CHARACTERISTICS } & $\begin{array}{c}\text { LAST VISIT } \\
\text { (n = 65) }\end{array}$ \\
\hline Age (years): Mean (SD) & $67(8.7)$ \\
\hline Sex: Women (\%) & $44(68 \%)$ \\
\hline Duration of RA (years): Mean (SD) & $17(7.7)$ \\
\hline Baseline Visit- Final Visit (years): Mean (SD) & $5.5(0.95)$ \\
\hline Dyslipidemia: Yes (\%) & $47(72 \%)$ \\
\hline High blood pressure: Yes (\%) & $40(62 \%)$ \\
\hline Diabetes Mellitus: Yes (\%) & $12(18 \%)$ \\
\hline Smoking: Yes (\%) & $17(26 \%)$ \\
\hline Obesity: Yes (\%) & $26(40 \%)$ \\
\hline Total Cholesterol (mg/dl): Mean (SD) & $186(42.4)$ \\
\hline Waist circumference: Yes (\%) (0'>102 cm, \&>88 cm) & $42(69 \%)$ \\
\hline LDL- Cholesterol (mg/dl): Mean (SD) & $104(34.6)$ \\
\hline Atherogenic index: Mean (SD) & $3.37(0.88)$ \\
\hline Systolic blood pressure (mmHg): Mean (SD) & $136(14.2)$ \\
\hline Diastolic blood pressure (mmHg): Mean (SD) & $80(8.2)$ \\
\hline
\end{tabular}

Results: The results of the 65 patients at the last visit are shown in table 1 . The average age was of 67 years, with a disease duration of 17 years and high prevalence of dyslipidemia (72\%), HTA (62\%) and obesity (40\%).

Data obtained at the basal and last visit (with an average difference of 5.5 years) were compared in table 2. At last follow-up none of the smokers at baseline had discontinued smoking. There were no significant differences in the body mass index (BMI) average. Nevertheless, there was increase in the number of patients with a $\mathrm{BMI}<30(36 \%$ vs $31 \%)$. Only a few patients claimed to go on any type of diet $(20 \%)$, although the majority $(88 \%)$ had classic CV risk factors (DM, HTA obesity or dyslipidemia) at baseline that required adequate diet regime. Yet, more than half of the patients $(62 \%$ ) made regular exercise ( $>3$ times/week) and did not have a sedentary lifestyle (50\%), despite having a mean age of 67 years and long disease duration at baseline. Poor control of diabetes mellitus (DM), was found in more than half of the patients.

Abstract AB0384 - Table 2. Variables assessed at baseline visit and last visit of 65 patients with RA and very high CV risk.

\begin{tabular}{|l|c|c|}
\hline \multicolumn{1}{|c|}{ Variables } & $\begin{array}{c}\text { BASALINE VISIT } \\
(\mathbf{n}=65)\end{array}$ & $\begin{array}{c}\text { LAST VISIT } \\
(\mathbf{n}=65)\end{array}$ \\
\hline Smoking: Yes (\%) & $17(26 \%)$ & $17(26 \%)$ \\
\hline Discontinued smoking: Yes (\%) & - & 0 \\
\hline Obesity: Yes (\%) & $24(37)$ & $26(40 \%)$ \\
\hline BMI: Mean (SD) & $28.2(5)$ & $28.6(5,5)$ \\
\hline BMI > 30: Yes (\%) & $22(36 \%)$ & $19(31 \%)$ \\
\hline Diet: Yes (\%) & - & $12(20 \%)$ \\
\hline Exercise: Yes (\%) & - & $38(62 \%)$ \\
\hline Sedentarism: Yes (\%) & - & $30(50 \%)$ \\
\hline Diabetes Mellitus (DM): Yes (\%) & $6(9 \%)$ & $12(18 \%)$ \\
\hline DM. Treatment: Yes (\%) & - & $6(50 \%)$ \\
\hline DM. Higher Glycosylated Hemoglobin: Yes (\%) & - & $7(58 \%)$ \\
\hline
\end{tabular}

Conclusions: Despite having been informed on the high risk of $\mathrm{CV}$ events, patients with RA included in the category of very high CV risk performed poor long-term control of factors that include a healthy lifestyle.

Disclosure of Interest: None declared

DOI: 10.1136/annrheumdis-2018-eular.4207

\section{AB0385 COMPARATIVE ASSESSMENT OF BMD IN PRE-, POSTMENOPAUSAL WOMEN AND MAN WITH RHEUMATOID ARTHRITIS (RA)}

\section{O. Garmish, A. Romanovskyi, T. Orlyk. Institute of Cardiology, Kyiv, Ukraine}

Background: RA doubles the risk of hip and vertebral fractures, regardless of the use of GCs, and disease activity is consistently associated with low BMD. But now, it is not clearly identified predictors of the individual risk of bone loss depends on sex and menopausal status patients with RA

Objectives: To compare BMD in man, pre- and postmenopausal women with RA Methods: The study was performed on 145 patients: 117 women (mean age 45.4 \pm 13.0 years, mean disease duration $9.7 \pm 7.7$ years, $41 \%(n=48)$ postmenopausal and 28 man (mean age $46.4 \pm 16.9$ years, mean disease duration $4.2 \pm 4.1$ years) with RA. $91.6 \%$ have moderate/high disease activities by DAS $28.68 .4 \%$ women and $64.3 \%$ men received prednisolon $\leq 10 \mathrm{mg} /$ day more than 3 month, $87 \%$ of patients received MTX. BMD was measured in 3 part of the skeleton: hip, lumbar spine, distal part of forearm. Female patients were divided in two groups by menopause: premenopausal (PreM) in mean age $36.9 \pm 9.3$ years and postmenopausal $(P M)$ in the mean age $57.6 \pm 5.9$ years.

Results: BMD was decreased in $44.5 \%$ of women and $42.9 \%$ of man. BMD of hip, lumbar spine, distal part of forearm were respectively decreased in $26.1 \%$ $26.1 \%, 18.8 \%$ PreM women and $66.7 \%, 70.8 \%, 79.2 \%$ PM women. $39.3 \%$ of man had decrease BMD in the hip and $42.8 \%$ - in the lumbar spine. In women the age was strong associated with BMD decrease, in man no association with age was found. In PreM women was not found association between BMD, disease duration, DAS28 and X-ray changes in hands and feet, only cortical index was correlated with BMD in all part of the skeleton. In PM women the disease duration was negatively correlated with BMD in total hip and forearm, in men - with BMD in lumbar spine and hip neck $(p<0.01)$. It was found association between BMD and $X$ ray stage by Steinbrocker in PM women and man. DAS28 was strong associated with low hip and forearm BMD in PM women and low spine BMD in men. According to dispersion analysis PM women with III-IV X-ray stages has significantly lower BMD in the hip (total: $Z=2.16, p=0.04$; neck: $Z=2.61, p=0.01$ ) and medium part of forearm $(Z=2.92, p=0.001)$. Man had significantly lower $B M D$ in all part of the skeleton since II X-ray stage $(p<0.001)$ and negative correlation between BMD and presence of erosion.

Conclusions: A sexual differences in BMD loss was observed in different parts of the skeleton. In man the most affected part of the skeleton was spine and BMD changes were more likely to PreM women, had high association with disease activity by DAS28 and presence of erosion, and no association with age. In PreM women only cortical index had high predictive value for decrease BMD in all parts of the skeleton. Age, disease deration, duration of menopause, DAS28 and x-ray changes in hand and feet was strong associated with decreased BMD in the hip and forearm in PM women.

$\sqrt{10}$

N 